\title{
Andrei Nikolaevich Kolmogorov
}

\author{
V. M. TIHOMIROV \\ Department of Mathematics, Moscow State University, \\ 117234 Moscow, USSR
}

On the 20th of October 1987 one of the greatest scholars of our time, Andrei Nikolaevich Kolmogorov, passed away.

A. N. Kolmogorov was born in 1903. His mother died in childbirth, his father did not take part in his upbringing, but in his childhood Kolmogorov was surrounded by love, care and attention. His mother's sister saw to his education and his early childhood was passed at her father's estate, his grandfather, a former big landowner. Those surrounding the boy tried to instill in him a love of knowledge, books and nature. 'I discovered the pleasure of mathematical discoveries early, at the age of five or six. I noticed the relations $1=1^{2}, 1+3=2^{2}, 1+3+5=3^{2}, 1+3+5+7=4^{2}$, etc.' At home, where the boy grew up, his relatives organized a small school in the spirit of the new pedagogical ideas of that time. They 'published' a journal, 'Spring Swallows', in which the five year old boy 'oversaw' the mathematical section. He published his discovery in this journal.

When the boy turned six years old, he moved to Moscow with his aunt. He was accepted into a Moscow gymnasium considered one of the most progressive of that time. The gymnasium was organized by a group of intelligentsia with a radical bent. The fact that along with normal studies many interesting pedagogical experiments were conducted differentiated it from most of the gymnasia of the time.

During his studies, the boy's circle of interests were exceptionally broad. He studied biology and physics seriously. At the age of 14 he studied higher mathematics from an encyclopedia, was interested in chess and was absorbed by social problems (and, in particular, wrote a constitution for a utopian island government commune, where principles of higher justice would be realized). In 1917 he participated in the voting for the Constituent Assembly and studied history in depth. He was deeply fascinated by history. He later recounted 'My first scholarly talk, which I gave at the age of 17 at Moscow University, was a talk at the seminar of Professor C. V. Bahruschin on landowners in Novgorod'. At this point Kolmogorov made a discovery which was recognized by Bahruschin, one of the leading Russian historians of the time. The youth asked the professor if he could publish his result. He received the following answer: 'Well, you don't say young man. You offer only one proof. For a historian that is not enough. One needs at least five proofs'. It is possible that the disappointment of that moment influenced his future fate, he preferred to become a mathematician where just one proof is enough.

He still did not immediately decide to tie his future to mathematics. While at Moscow University, he also enrolled (in 1920) at the Metallurgical Department of 
the Chemical-Technological Institute, since he wanted to pursue useful engineering. But soon he remained only at the Physics-Mathematics Department of the University and has remained connected with the University ever since.

At the University he was quickly engulfed in a creatively uplifting atmosphere. He attended the lectures of the leading scholars, in particular his future teacher Nikolai Nikolaevich Lusin, and engaged in lively scholarly contact with the students of Lusin, Pavel Sergeevich Alexandrov and Pavel Samuilovich Urysohn, who would in the future play an important role in the development of topology.

Students recount that once (in 1920), while listening to Lusin, the first year student refuted one hypothesis of the lecturer. He received some attention for the first time at the mathematical circle at which he presented this. Urysohn suggested to Andrei Nikolaevich that he should be his student. Kolmogorov began to build a general theory of operations on sets wanting to move it forward from the state in which it was left by Alexandrov and Suslin. In February 1922 he wrote a long paper on this subject. He participated in the seminar of V. V. Stepanov on trigonometric series. There he solved one of the problems suggested by Lusin and after that Lusin 'with some solemnity' (as A. N. Kolmogorov put it) offered to make him his student.

In the summer of 1922 (paper dated 6.2.22) Andrei Nikolaevich achieved an outstanding result: he constructed an everywhere diverging Fourier series. This result immediately gained him worldwide attention. We can consider this point to be the beginning of his incomparable creative path.

In 1925 Kolmogorov graduated from Moscow University and became Lusin's graduate student. Kolmogorov's influence on probability theory can be dated from the same year. In 1929 he took his first long cruise on the Volga with Pavel Sergeevich Alexandrov, marking the beginning of a friendship, which lasted to the last days of Pavel Sergeevich's life (P. Alexandrov passed away on November 16, 1982). In 1935 A. N. Kolmogorov and P. S. Alexandrov acquired, not far from Moscow, a house on the banks of the river Klyazma, where, for the most part, their creative life flowed. In the following fifty years several generations of mathematicians came to know this house.

In 1931 Kolmogorov became a Professor of Moscow University. In 1939 he was made a Full Member of the Academy of Sciences of the USSR. At the University Andrei Nikolaevich founded the Probability Theory Kafedra (translator's note: Kafedra = Sub-faculty) (he headed this Kafedra from 1938 until 1966), the Laboratory of Probability and Statistical Methods (he headed it from 1966 until 1976), the Department of Mathematical Statistics (which he headed from 1976 until 1980). From 1980 until 1987 he headed the Kafedra of Mathematical Logic. Kolmogorov was the Director of the Institute of Mathematics at MGU (Moscow State University) from 1933 to 1939 and again from 1951 until 1953. From 1954 to 1958 he was the dean of MGU's Mechanics-Mathematics Department.

A. N. Kolmogorov was the Secretary-Academician of the Physics-Mathematical Sciences Section of the Academy of Sciences (1939-42), he headed the Probability Theory Department of the Steklov Institute of Mathematics of the Academy of Sciences from 1939 to 1958, he headed the Laboratory of Atmospheric Turbulence 
of the Geophysics Institute of the Academy of Sciences from 1946 to 1949. From December 1964 to December 1966 and from November 1973 to October 1985 Kolmogorov was the President of the Moscow Mathematical Society.

Kolmogorov was in Göttingen, Münich and Paris from June of 1930 through March of 1931 where he met with Hilbert, Carathéodory, E. Landau, P. Levi, Fréchet, Lebesque, Borel, H. Weyl and other leading mathematicians. In 1954 he was, for two months, a visiting Professor at Humboldt University in Berlin and in the spring semester of 1958 he was a Visiting Professor at the University of Paris.

A. N. Kolmogorov took part in the workings of the International Congresses of Mathematics in Amsterdam (1954), Stockholm (1962), Moscow (1966) and Nice (1970). Andrei Nikolaevich was given the honour of closing the Amsterdam Congress with a lecture which he gave on dynamical systems (J. von Neumann opened the Congress).

More than twenty learned societies have made Andrei Nikolaevich a member. He was a member of the Royal Netherlands Academy of Science (1963), the Royal Society of London (1964), the National Academy of Sciences of the United States of America (1967), the Paris Academy of Sciences (1968), and Honorary member of the Romanian Academy of Sciences (1965, corresponding-member since 1957), a foreign member of the Polish Academy of Sciences (1956), and an honorary member of the Hungarian Academy of Sciences (1965). He received honorary doctorates from the Universities of Paris, Stockholm, Warsaw and Budapest, the Calcutta Mathematical Society and the London Royal Statistical Society as well as others.

He was deemed worthy of the USSR State Prize (together with A. Ya. Khinchin in 1941), the Chebychev Prize of the USSR Academy of Sciences (together with B. V. Gnedenko in 1949), the Lenin Prize (together with V. I. Arnold in 1965), the Lobachevsky Prize (1986), the International Prize of the Bolzan Foundation (1963, at the same time other categories of the prize were received by Pope John XXIII, historian C. Morrison, biologist K. Frish and composer P. Hindemith), the Wolf Prize, a Gold Medal from the American Meteorological Society, the Helmholtz Medal, etc.

Such were the lofty heights reached by Andrei Nikolaevich Kolmogorov.

A. N. Kolmogorov holds an exclusive place in the world of mathematical science. The depth of Andrei Nikolaevich's scholarly horizon is unique, he stands with the classical scientists of yore in the diversity of his intellectual pursuits. A. N. Kolmogorov's results include both basic and fundamental results, ideas and complete definitive theorems. He has posed problems which have given a powerful impetus to new research.

The creative life of Andrei Nikolaevich lasted nearly fifty years. We will review it in ten year periods.

The first ten years - the twenties. Andrei Nikolaevich worked on, first of all, problems characteristic of the Lusin school of function theory (the theory of trigonometric and orthogonal series, operations on sets, measure theory and integration). But at the same time he also began a cycle of research on probability theory 
and wrote two papers on mathematical logic. Even at this stage, at the dawn of his creative life, Kolmogorov's work combined the creation of new concepts with the solution of difficult fundamental problems.

We note four theorems pertaining to harmonic analysis and probability.

(a) He constructed a function $x \in L_{1}([-\pi, \pi])$ whose Fourier series diverges everywhere. The result has a definitive character. Almost fifty years later Carleson and Hunt showed that if $x \in L_{p}([-\pi, \pi]), p>1$ then its Fourier series converges almost everywhere.

(b) The proof that the Hilbert transformation is, as we would say today, of weak type $(1,1)$. This means that if $x \in L_{1}([-\pi, \pi])$ and $\tilde{x}$ is the conjugate complex function, then Meas. $\{t:|\tilde{x}(t)| \geq \alpha\} \leq\|x\|_{1} \alpha^{-1}$. This result is a cornerstone of harmonic analysis. It was the basis of future research in the work of M. Riesz, Marcinkiewicz, Zygmund, Calderon, etc.

(c) The finding of necessary and sufficient conditions for the law of large numbers - a problem posed by P. L. Chebychev and A. A. Markov.

(d) The finding of the wide conditions for the law of iterated logarithms.

Along with these, the general theory of operations on sets was developed, giving a universal approach to our fundamental understanding of measure and integration. (A. N. Kolmogorov repeatedly expressed the hope that his research of the twenties and thirties on measure theory and integration would take its proper place in the further development of function theory). Besides all this, classical logic was immersed into intuitionistic logic (that is, logic without the law of the excluded middle) and yet it was shown that the application of the law of the excluded middle, by itself, cannot lead to a contradiction. In the second work it was shown that intuitionistic logic is tractable, as is constructive logic. His works of this period on mathematical logic long ago entered the foundation of this subject.

The second ten year period was the thirties. This was, perhaps, one of the two most fruitful ten year periods. Of course, the most well known cycle of Kolmogorov's works on probability theory belong to this period, but he also worked on geometry, functional analysis, algebraic topology, general topology, approximation theory, differential equations and mathematical analysis. New concepts, fundamental ideas, primary results and the formulation of new problems prevailed in this period.

In 1933 Kolmogorov's monograph 'Fundamental Concepts of Probability Theory' appeared, which along with the works of Ya. Bernoulli and Laplace became a real classic in probability theory. This monograph marked a new stage in the development of probability theory as a mathematical science. Axiomatic probability theory was partially developed in it.

In 1933 the wonderful paper 'Analytic Methods in Probability Theory' appeared. There the theory of Markov processes was developed and Markov processes and parabolic differential equations were tied for the first time. Forward and backward Kolmogorov equations were introduced in this work. Earlier forward equations had, in part, appeared in the works of Einstein, Fokker, Planck, Smoluchovsky and other leading physicists. The theory of Markov processes became a powerful scientific implement after the paper of Kolmogorov. 
At the end of this ten year period, the theory of random stationary processes was, in fact, developed by A. N. Kolmogorov. Somewhat later N. Wiener independently arrived at the same results which he considered among his highest achievements. But besides all this, Andrei Nikolaevich Kolmogorov was (on a level with von Neumann) one of the pioneers of the theory of linear topological spaces, where he had a fundamental result-a criterion for the existence of a norm in a linear topological space. He was the source of topological geometry, having described continuous projective geometry on local fields. He, concurrently with Alexander, a leading American topologist, arrived at one of the most important understandings of algebraic topology which transformed all of topology, namely the understanding of cohomology and the building of the theory of cohomological operations. He enriched approximation theory with the posing of new problems and with the introduction of new ideas. This served as a powerful impetus in the development of this area of analysis, in which hundreds would take part. He, along with I. G. Petrovski and N. C. Piskunov, developed the mathematical theory of stability of the running wave solution for diffusion equations with a non-linear right hand side (a bibliography of works on this theme would include over a thousand references). The theory of Markov chains with countable states was also developed and much else was done.

We note the following results:

(a) The construction of an open mapping which increases dimension.

(b) The proof of the fundamental theorem on the deviation of the empirical distribution function from the actual distribution function.

(c) The solution of Hadamard's problem on inequality for derivatives.

The forties were notable for the movement of interest toward applications. An important cycle of works appeared pertaining to turbulence theory. Kolmogorov's work transformed this field and greatly influenced all future development of this modern area of natural science. One of his well known concrete results is the 'law of two thirds'. The discovery of the law was an important stage in the development of the study of nature. It reads: in a turbulent flow the average of the square of the difference of the speed of two points at a distance $r$ from each other (average size) is proportional to $r^{2 / 3}$.

In these years Kolmogorov essentially developed ballistics, worked on biological problems, addressed problems in geology, meteorology, statistical control and crystallography.

In the sphere of mathematics he laid the foundation of the theory of branching processes and evolved the theory of interpolation and extrapolation of random sequences. In these years he wrote (together with B. V. Gnedenko) the classic work on limiting distributions for sums of independent random variables.

Among all his concrete results we note one theorem (left unpublished by A. N. Kolmogorov) on the completeness of irreducible representations of groups.

The next ten years, the fifties, were characterized by an unusual rise in creative energy. Here again, as in his younger days, the creation of important new concepts is combined with the solution of long standing and fundamental problems. 
Here we must point to, first of all, a cycle of works on classical mechanics. A. N. Kolmogorov presented an overview of these results at the above mentioned Amsterdam Congress. The problem of the evolution of orbits in the three body problem can be traced back to Newton and Laplace. Poincare called the problem of the behavior of Hamiltonian systems under small perturbations of the Hamiltonian function 'the fundamental problem of dynamics'. This problem was solved in the general situation in Kolmogorov's work for most initial conditions. The methods of proof developed in this work played a great role in classical analysis, mechanics and other areas of natural science. Later it was further developed in the work by V. I. Arnold and J. Moser and became known under the name KAM-theory (the theory of Kolmogorov-Arnold-Moser).

In these years Kolmogorov achieved the following fundamental result: any continuous function on an $\boldsymbol{n}$-dimensional cube (any $n$ ) can be represented as a superposition of continuous functions of three variables. This reduces Hilbert's 13th problem to the concrete problem of representing functions on universal trees in three dimensional space. This last problem was solved by V. I. Arnold (solved under the guidance of A. N. in third year student work), thus refuting the hypothesis of Hilbert.

Later, A. N. Kolmogorov obtained the following definitive result: a continuous function on a cube of any dimension is representable as a superposition of continuous functions and the operation of addition.

Using ergodic theory Kolmogorov introduced a new metric invariant, the entropy of dynamical systems. On the one hand, this solved the classic problem about the non-isomorphism of Bernoulli automorphisms, and on the other hand, a completely new field of research was opened, later leading to chaos theory.

Apart from these exceptionally important works, A. N. Kolmogorov found wonderful new approaches to algorithm theory, formulated the basic principles of enumeration theory, wrote a fundamental work on statistics (unbiased estimates), proved the uniform limit theorem for sums of independent summands (A. N. was very proud of this result), introduced the idea of entropy and thus initiated a new aspect in approximation theory, made an important contribution to information theory and proved that analytic function spaces with a different number of variables cannot be isomorphic.

The sixties - the last ten year period when Andrei Nikolaevich actively studied mathematics proper. In this period he started to turn more often to pedagogical problems. Moreover, he often works on problems of prosody.

At the last stage of his creative life Andrei Nikolaevich Kolmogorov advanced the grandiose program of the simultaneous study of complexity, the stochastic properties of deterministic processes and regular indeterminacy of random processes and phenomena. The experience of practically all his creative life is concentrated in this program. In his articles of 1965 and 1969 published in 'Problemi Peredachi Informatzi' (Problems of Information Transfer) Andrei Nikolaevich laid the foundation of a new field of knowledge, the algorithmic theory of information, and gave a new approach to the logical foundations of Probability Theory.

Such, in general terms, are the heights of his creative biography. 
Andrei Nikolaevich has influenced many scholars. His own schools of thought must be pointed out. Nearly sixty graduate students worked under Kolmogorov's supervision. Many of them later became leaders of various scholarly schools. The spectra of these schools is tremendous. We list several of Kolmogorov's students and indicate the field they studied under his supervision:

Probability theory: Yu. V. Prohorov (Academician Academy of Sciences, USSR), A. A. Borvkov (corresponding member, Academy of Sciences, USSR), B. A. Sevastyanov (corr. Mem. A.S.), B. V. Gnedenko (acad. Ukranian Academy of Sciences), S. H. Sirazdinov (acad. Uzbekistan Academy of Sciences), Professors P. L. Dobrushin, V. M. Zolotarev, L. D. Meshalkin, Yu. K. Belyaev, Ya. A. Rozanov, A. N. Shiryaev, etc.

Mathematical Statistics: L. N. Bolshev (corr. mem. A.S.).

Dynamical Systems, Mechanics: V. I. Arnold (corr. mem. A.S.) Professors V. M. Alexseev, Ya. G. Sinai.

Functional Analysis: I. M. Gelfand (acad. A.S.), Professor S.F. Fomin.

Approximation theory: S. M. Nikolski (acad. A.S.), Professor V. M. Tihomirov, V. D. Erohin, etc.

Applied Physics, Mechanics: M. D. Millionchikov (acad. A.S.).

Atmospheric Physics: A. M. Obukhov (acad. A.S.), Professors A. M. Yaglom, G. I. Barenblatt.

Oceanology: A. C. Monin (corr. Mem. A.S.).

Mathematical Logic: A. I. Malchev (acad. A.S.), Professor V. A. Uspenski.

Cybernetics: V. C. Mihalevich (acad. A.S.).

Information Theory: Professors M. C. Pinsker, L. A. Bassaligo.

It seems probable that no one other person has overseen such a diverse collection of schools of thought.

During the last twenty five years of his life A. N. Kolmogorov dedicated himself foremost to the enlightenment of the education of middle schools.

In the arts he was especially knowledgeable on ancient Russian architecture, all of Russian poetry, worldwide sculpture and painting. He spent much time daily (especially in his last years) listening to music.

Andrei Nikolaevich liked to travel. In the span of fifty years he travelled annually, with friends and later with many of his students. He was especially drawn to the Russian North, the Volga, the Crimea and the Caucasus. Nature endowed him with excellent health, which gave him a chance to lead an active sports life, drawing the young along with him. He was an expert crosscountry skier, he could stand atop his skis for twelve hours while skiing large distances. He also engaged in swimming and downhill skiing.

All his life was dedicated to the development of mankind's culture in the broadest possible sense. The power of his thought, and the broad, diverse horizon of his knowledge of science, politics and arts was stupendous.

Andrei Nikolaevich can be numbered among those incomparable geniuses with shining personalities which give one the strength to live just by the mere fact of their existence. 